\title{
Use of Ultra-Deep Sequencing in a Patient with Tuberculous Coxitis Shows Its Limitations in Extrapulmonary Tuberculosis Diagnostics: A Case Report
}

This article was published in the following Dove Press journal:

Infection and Drug Resistance

\author{
Chi Zhang ${ }^{1} *$ \\ Tao $\mathrm{Hu}^{2, *}$ \\ Leshan Xiu' \\ Yamei $\mathrm{Li}^{\mathrm{I}}$ \\ Junping Peng (iD) \\ 'NHC Key Laboratory of Systems \\ Biology of Pathogens, Institute of \\ Pathogen Biology, Chinese Academy of \\ Medical Sciences \& Peking Union Medical \\ College, Beijing, People's Republic of \\ China; ${ }^{2}$ Department of Neurosurgery, \\ Shanxi Provincial People's Hospital, \\ Taiyuan, People's Republic of China \\ *These authors contributed equally to \\ this work
}

\begin{abstract}
Unbiased metagenomic next-generation sequencing (mNGS) has been widely applied in clinical microbiology for infectious disease diagnostics. Some successful applications in central nervous system infections and respiratory tract infections have proved it to be a promising tool for pathogen detection. Here, mNGS was used to confirm the infectious etiology in a case of tuberculous coxitis, which is a common type of extrapulmonary tuberculosis (TB). A 77-year-old Asian male presented with hip pain and fever was diagnosed with smear- and culture-negative tuberculous coxitis based on the evidence of imaging and interferon- $\gamma$ release assay. At the same time as the anti-TB therapy started, the patient underwent joint clearance surgery. Whole blood and synovial fluid sampled from surgery were kept for bacteriological confirmation using mNGS with high sequencing depth. However, only two paired-end reads from synovial fluid samples were identified as Mycobacterium tuberculosis. Before leaving hospital, the patient was still receiving antiTB treatment and began to recover. Considering the obvious escalation of the cost and analysis time as the depth of sequencing increases, although we got a positive result here, the scarce number of reads obtained through ultra-deep sequencing indicates its limitations in extrapulmonary tuberculosis.
\end{abstract}

Keywords: extrapulmonary tuberculosis, metagenomics, molecular diagnostic techniques, Mycobacterium tuberculosis

\section{Introduction}

Unbiased metagenomic next-generation sequencing (mNGS) has been widely applied in clinical microbiology. Taking advantage of its comprehensiveness, mNGS has been successfully used to identify infrequent or even novel pathogens. Moreover, for fastidious organisms, such as mycobacteria or fungi, the turnaround time of mNGS outperforms traditional culture. Some successful applications in central nervous system infections and respiratory tract infections have proved it to be a promising diagnostic tool for infectious diseases. ${ }^{1}$ Different from cases of acute infections, where the pathogen load is usually high, some diseases present a paucibacillary nature, which makes it difficult to establish a diagnosis. Such is the case with extrapulmonary tuberculosis. Here, we reported a case of using mNGS to identify the infectious agent from tuberculous coxitis and evaluated the performance of mNGS.
Correspondence: Junping Pen

of Pathogens, Institute of Pathogen Biology, Chinese Academy of Medical Sciences \& Peking Union Medical College, No. 6, Rongjing East Street, BDA, Beijing, People's Republic of China

$\mathrm{Tel} / \mathrm{Fax}+861067878493$

Email pengjp@hotmail.com 


\section{Case Report}

A 77-year-old Asian male presented with a 2-month duration of pain and swelling in the medial aspect of the left thigh and fever. Before referring, two aspirations were performed by a community hospital, but the pain recurred after temporary relief. The patient's medical history was noteworthy for degenerative lumbar disk disease and he had undergone inguinal herniorrhaphy 16 years prior. He was not on any long-term medication. At the time of admission, the patient had been suffering aggravating pain and lameness. Upon physical examination, the movement of the patient's left hip joint was restricted and the Patrick's test was positive. The patient had a temperature of $37.3^{\circ} \mathrm{C}$ without chills. The patient's bloodwork was not clinically significant except hemoglobin $118 \mathrm{~g} / \mathrm{L}$ (reference: $120-160 \mathrm{~g} / \mathrm{L}$ ), erythrocyte sedimentation rate (ESR) $60 \mathrm{~mm} /$ hour (reference: $0-15 \mathrm{~mm} /$ hour), and C-reactive protein $(\mathrm{CRP})>150 \mathrm{mg} / \mathrm{L}$ (reference: $0-5 \mathrm{mg} / \mathrm{L}$ ). Blood cultures tested for aerobic and anaerobic bacteria were negative. Magnetic resonance imaging (MRI) revealed a small amount of effusion in the left acetabular cavity. An increased T2 signal of the left iliopsoas was also revealed by MRI, indicating infection. No joint space narrowing was observed. Computed tomography (CT) showed spotted calcification in the upper and lower lobes of both lungs, which hinted at a history of pulmonary tuberculosis (TB). An interferon- $\gamma$ release assay (TSPOT. TB) was performed and the positive reaction indicated latent or active TB. The affected hip was assumed to be the result of prior pulmonary infection with Mycobacterium tuberculosis (MTB), which reached the joint via hematogenous spread.

Based on this evidence, the patient began to receive anti-TB therapy with a combination of oral isoniazid, rifampicin, pyrazinamide, and ethambutol. Although the patient's pain was partially relieved and the MRI revealed a smaller lesion, his temperature continued to rise and the pain aggravated after five days of treatment. The patient then underwent joint clearance surgery and vacuum sealing drainage (VSD) to alleviate the symptoms. During the surgery, granulomatous inflammation was found and caseous materials were removed from the femoral neck, femoral head, and joint capsule. The caseous materials and synovial fluid were collected for microbiological examination. Although a biopsy specimen showed histopathological features of mycobacterial arthritis, microscopy for acid-fast bacilli and culture were negative.

The patient's whole blood and synovial fluid were kept for bacteriological confirmation using mNGS with high sequencing depth to increase the sensitivity. Briefly, nucleic acids were extracted using the QIAamp DNA Mini Kit (Qiagen, Hilden, Germany) and libraries were constructed using the NEBNext Ultra DNA Library Prep Kit (NEB, Ipswich, MA, USA). Libraries were sequenced using an Illumina HiSeq2500 sequencer with paired-end reads of an average $150 \mathrm{bp}$ in length. In-house Perl scripts were used to filter low-quality reads and Bowtie 2 to remove reads with similarity to the human genome. The workflow and criteria used in this study were described previously. ${ }^{2}$ BLASTn was used to align the remainder against the NCBI non-redundant nucleotide database with an E-value $<10^{-5}$, and hits were analyzed and extracted by MEGAN 5 software. ${ }^{3}$ The postfiltering data sets have been stored in the NCBI Sequence Read Archive and are available from BioSample accession number SAMN13246068 and SAMN13246069 under BioProject PRJNA588503.

Sequencing the libraries from synovial fluid and whole blood yield 32,629,075 and 36,759,980 clean paired-end reads representing $9.79 \mathrm{~GB}$ and $11.03 \mathrm{~GB}$ of nucleotide data, respectively. Following quality control and eliminating human sequences, for the synovial fluid and whole blood sample, the filtered sequence data contain 53,887 and 109,198 reads, among which 681 and 144 reads yield alignments to bacterial sequences, respectively. Among all hits, only two paired-end reads from synovial fluid samples were identified as MTB. Remaining reads belonging to genera of Corynebacterium, Brevundimonas, Propionibacterium, Micrococcus, Kocuria, and Stenotrophomonasa account for the majority of the bacterial hits (Figure 1). Most of the viral reads belong to Torque teno virus (TTV), a member of the family Anelloviridae. Detailed proportions of each bacterial and viral taxa were listed in Supplementary materials (Table S1). Due to the low possibility of contamination with mycobacteria, the criterion for MTB being considered positive by mNGS was at least one read mapped to its genome. ${ }^{1,4}$ Meanwhile, except MTB, other identified bacteria are regarded as contaminants commonly found in laboratory environments, ${ }^{5}$ and furthermore, none of them should be classified as pathogen, because they were present at levels equivalent to the background. For viruses, infections with TTV are ubiquitous in human population and there are no convincing pieces of evidence showing that TTV is associated with any specific diseases. ${ }^{6}$ Considering all the above, we conclude that the results of mNGS can provide evidence of MTB infection.

After surgery, the patient was treated for a surgical site infection with isoniazid and the oral anti-TB therapy continued. Four weeks after surgery, MRI revealed a reduced 

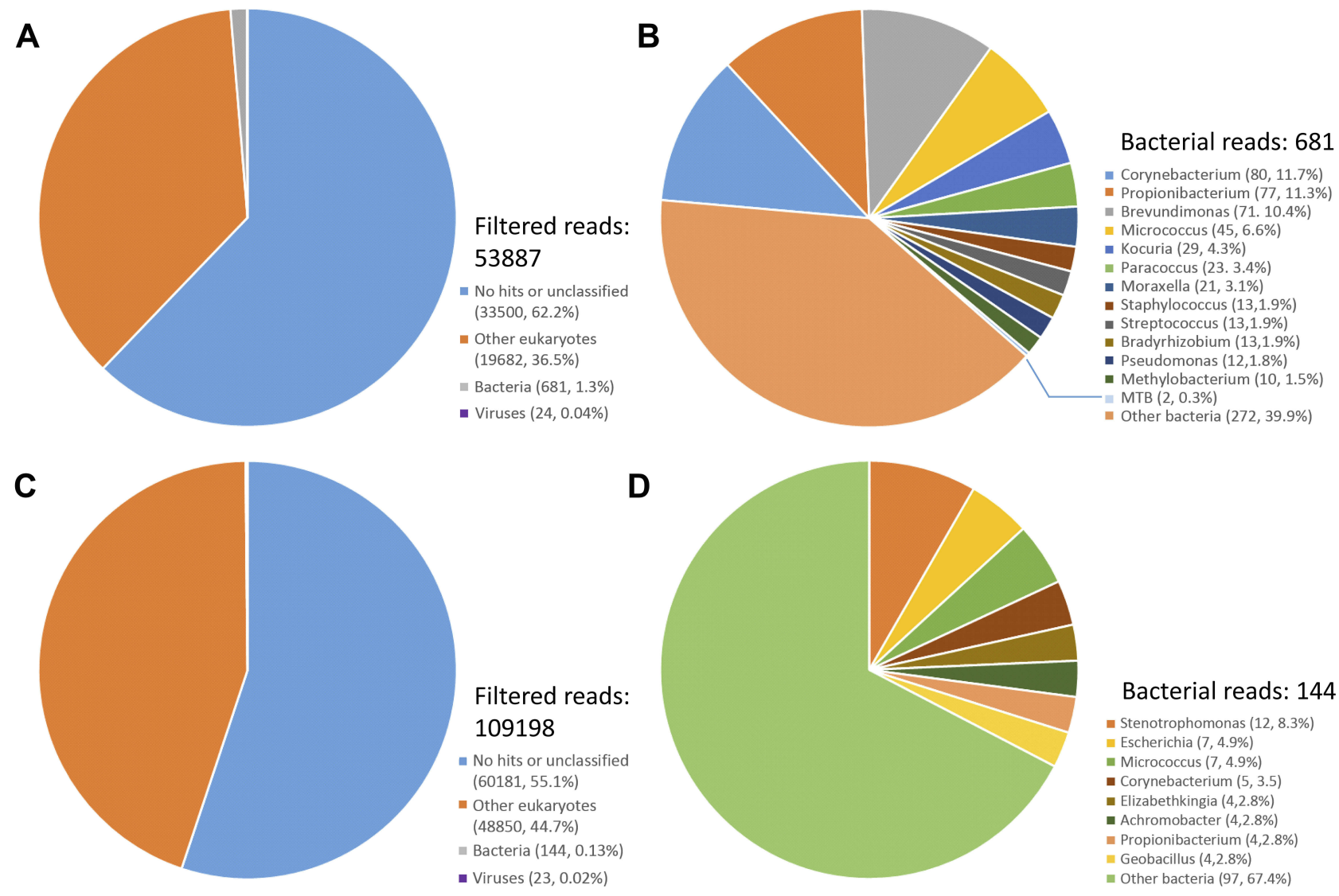

Figure I Taxonomic classification of reads from (A) filtered reads of the synovial fluid sample, (B) bacterial reads of the synovial fluid sample, (C) filtered reads of the whole blood sample, and (D) bacterial reads of the whole blood sample.

range of the lesion compared to before the surgery and the CRP gradually decreased. The patient's hip pain was relieved and temperature stabilized. At the patient's request, he was allowed to leave the hospital. A timeline showing the patient's entire clinical course and the results of important tests is provided in Supplementary materials (Figure S1).

\section{Discussion}

Here, mNGS was employed in a case of osteoarticular TB to confirm the presence of MTB, but our findings illustrated the limitations of mNGS. Previous studies have demonstrated that extrapulmonary $\mathrm{TB}$ is a paucibacillary disease, and the verification rate through culture is low. ${ }^{7,8}$ In this case, only two paired-end reads were acquired by ultra-deep sequencing, which is in accordance with the paucibacillary nature of the disease. This means to get an informative result, a higher sequencing depth is needed. According to the criterion, although a positive result was obtained here, the huge sequencing costs and analysis time resulting from the ultra-deep sequencing were far beyond many hospitals could bear. Similar dilemma was seen in two other cases of extrapulmonary tuberculosis. In a case of osteoarticular tuberculosis, six reads of MTB were obtained from 32,990,757 reads, and in a case of brain lesions, 15 reads of MTB were obtained from 13,990,253 reads. ${ }^{9,10}$ Both of the studies used ultra-deep sequencing to identify the existence of MTB, but MTB-related reads only covered a tiny proportion of the total sequencing data. As a result, the role mNGS plays in extrapulmonary tuberculosis is inferior to its role in acute infectious diseases.

In addition to the straightforward way of increasing the sequencing depth, there are other approaches to enriching MTB in the samples, thereby increasing the chances of being detected. For example, sequencing mycobacteria from early liquid cultures is faster and cheaper than routine MTB diagnostic workflows in predicting species and drug susceptibility. ${ }^{11}$ Moreover, biotinylated RNA baits can be used to enrich MTB DNA prior to direct sequencing of clinical samples, making whole-genome sequencing available. ${ }^{12}$ Although detecting MTB from extrapulmonary 
tuberculosis samples requires a higher depth, the sensitivity of mNGS can be improved through appropriate optimization, thereby reducing the analysis time and computational resources.

Xpert has been suggested by WHO for diagnosis of TB and rifampicin-resistance, and its performance on extrapulmonary tuberculosis has been assessed by several studies. For osteoarticular TB, Xpert has been shown to be a sensitive method, with sensitivities ranging from $66.7 \%$ to $85.2 \%$, and its application could increase the ratio of confirmed cases. ${ }^{13-15}$ In addition, investigating the expression of some RNA signatures from blood samples is a promising molecular test. This method can identify individuals with latent tuberculosis infection who are at risk of progression to active tuberculosis, severing as an important test to achieve targeted treatment of latent tuberculosis infection. $^{16,17}$

Compared to pulmonary $\mathrm{TB}$, extrapulmonary $\mathrm{TB}$ is less valued and does not receive reasonable attention, though it continues to impose a heavy burden on public health. Extrapulmonary TB commonly occurs in the pleura, lymph nodes, bones and joints, genitourinary tract, and meninges, among which osteoarticular TB accounts for approximately $10 \%$ of all extrapulmonary TB cases. ${ }^{8,18}$ TB of the hip is the second most common type of osteoarticular TB, behind TB of the spine. ${ }^{19,20}$ In the early stage of the disease, the symptoms and radiological features are usually non-specific, but timely diagnosis is important, as the delay can lead to irreversible destruction of the hip. Finding MTB in body fluid or tissue by either culture or smear microscopy is the gold standard in the diagnosis of osteoarticular TB. However, as this case indicates, the slow growth of MTB and paucibacillary nature of the disease make identifying the pathogen by routine microbiological techniques or even mNGS rather challenging. Therefore, a definitive diagnosis of smearand culture-negative osteoarticular TB should be established based on a composite reference standard including conventional laboratory examinations, radiological signs, molecular tests, and the patient's response to anti-TB treatment.

\section{Ethical Statement}

The study was performed in accordance with the recommendations of national ethics regulations and approved by the Institutional Review Board of the Institute of Pathogen Biology. Written informed consent was given by the patient to publish the information in this case report.

\section{Data Sharing Statement}

Most of the data generated or analyzed are available in the current study. If necessary, all genomic data could be available from the corresponding author on reasonable request.

\section{Acknowledgments}

This work was supported by the Ministry of Science and Technology of the People's Republic of China (2014ZX10004001).

\section{Author Contributions}

All authors contributed to data analysis, drafting or revising the article, gave final approval of the version to be published, and agreed to be accountable for all aspects of the work.

\section{Disclosure}

The authors declare that they have no conflict of interest.

\section{References}

1. Simner PJ, Miller S, Carroll KC. Understanding the promises and hurdles of metagenomic next-generation sequencing as a diagnostic tool for infectious diseases. Clin Infect Dis. 2018;66(5):778-788. doi:10.1093/cid/cix881

2. Yang J, Yang F, Ren L, et al. Unbiased parallel detection of viral pathogens in clinical samples by use of a metagenomic approach. J Clin Microbiol. 2011;49(10):3463-3469. doi:10.1128/JCM.00273-11

3. Huson DH, Auch AF, Qi J, Schuster SC. MEGAN analysis of metagenomic data. Genome Res. 2007;17(3):377-386. doi:10.1101/gr.5969107

4. Miao Q, Ma Y, Wang Q, et al. Microbiological diagnostic performance of metagenomic next-generation sequencing when applied to clinical practice. Clin Infect Dis. 2018;67(suppl_2):S231-S240. doi:10.1093/cid/ciy693

5. Salter SJ, Cox MJ, Turek EM, et al. Reagent and laboratory contamination can critically impact sequence-based microbiome analyses. BMC Biol. 2014;12:87. doi:10.1186/s12915-014-0087-z

6. Shulman LM, Davidson I. Viruses with circular single-stranded DNA genomes are everywhere! Аnпи Rev Virol. 2017;4(1):159-180. doi:10.1146/annurev-virology-101416-041953

7. Chen ST, Zhao LP, Dong WJ, et al. The clinical features and bacteriological characterizations of bone and joint tuberculosis in China. Sci Rep. 2015;5:11084. doi:10.1038/srep11084

8. Sandgren A, Hollo V, van der Werf MJ. Extrapulmonary tuberculosis in the European Union and European Economic Area, 2002 to 2011. Euro Surveill. 2013;18(12):20431

9. Huang Z, Zhang C, Hu D, et al. Diagnosis of osteoarticular tuberculosis via metagenomic next-generation sequencing: a case report. Exp Ther Med. 2019;18(2):1184-1188. doi:10.3892/etm.2019.7655

10. Salzberg SL, Breitwieser FP, Kumar A, et al. Next-generation sequencing in neuropathologic diagnosis of infections of the nervous system. Neurol Neuroimmunol Neuroinflamm. 2016;3(4):e251. doi:10.1212/NXI.0000000000000251

11. Pankhurst LJ, Del Ojo Elias C, Votintseva AA, et al. Rapid, comprehensive, and affordable mycobacterial diagnosis with whole-genome sequencing: a prospective study. Lancet Respir Med. 2016;4 (1):49-58. doi:10.1016/S2213-2600(15)00466-X 
12. Brown AC, Bryant JM, Einer-Jensen K, et al. Rapid whole-genome sequencing of mycobacterium tuberculosis isolates directly from clinical samples. J Clin Microbiol. 2015;53(7):2230-2237. doi:10.1128/JCM. 00486-15

13. Gu Y, Wang G, Dong W, et al. Xpert MTB/RIF and GenoType MTBDRplus assays for the rapid diagnosis of bone and joint tuberculosis. Int $J$ Infect Dis. 2015;36:27-30. doi:10.1016/j. ijid.2015.05.014

14. Li Y, Jia W, Lei G, Zhao D, Wang G, Qin S. Diagnostic efficiency of Xpert MTB/RIF assay for osteoarticular tuberculosis in patients with inflammatory arthritis in China. PLoS One. 2018;13(6):e0198600. doi:10.1371/journal.pone.0198600

15. Wang G, Dong W, Lan T, et al. Diagnostic accuracy evaluation of the conventional and molecular tests for Spinal Tuberculosis in a cohort, head-to-head study. Emerg Microbes Infect. 2018;7(1):109. doi:10.1038/s41426-018-0114-1
16. Zak DE, Penn-Nicholson A, Scriba TJ, et al. A blood RNA signature for tuberculosis disease risk: a prospective cohort study. Lancet. 2016;387(10035):2312-2322. doi:10.1016/S0140-6736(15)01316-1

17. Levin M, Kaforou M. Predicting active tuberculosis progression by RNA analysis. Lancet. 2016;387(10035):2268-2270. doi:10.1016/ S0140-6736(16)00165-3

18. Peto HM, Pratt RH, Harrington TA, LoBue PA, Armstrong LR. Epidemiology of extrapulmonary tuberculosis in the United States, 1993-2006. Clin Infect Dis. 2009;49(9):1350-1357. doi:10.1086/ 599192

19. Babhulkar S, Pande S. Tuberculosis of the hip. Clin Orthop Relat Res. 2002;398:93-99. doi:10.1097/00003086-200205000-00013

20. Johansen IS, Nielsen SL, Hove M, et al. Characteristics and clinical outcome of bone and joint tuberculosis from 1994 to 2011 a retrospective register-based study in Denmark. Clin Infect Dis. 2015;61(4):554-562. doi:10.1093/cid/civ326

\section{Publish your work in this journal}

Infection and Drug Resistance is an international, peer-reviewed openaccess journal that focuses on the optimal treatment of infection (bacterial, fungal and viral) and the development and institution of preventive strategies to minimize the development and spread of resistance. The journal is specifically concerned with the epidemiology of antibiotic resistance and the mechanisms of resistance development and diffusion in both hospitals and the community. The manuscript management system is completely online and includes a very quick and fair peerreview system, which is all easy to use. Visit http://www.dovepress.com/ testimonials.php to read real quotes from published authors 